\title{
Report on the analyses and the applications of a large-scale news video archive: NII TV-RECS
}

\author{
Ichiro IDE \\ Graduate School of Information Science, Nagoya Univ. / National Institute of Informatics
}

\begin{abstract}
This paper summarizes the work done in the last twelve years and more, on the structuring and the application of a large-scale digital news video archive: TV-RECS constructed at the National Institute of Informatics. The applications include those that facilitate the exploring and the browsing of the archive, and also the recompilation of its contents for the generation of new video contents.
\end{abstract}

\section{KEYWORDS}

Video archive, news, structuring, browsing, recompilation

\section{Introduction}

\subsection{Background}

Recent advance of storage technology has allowed us to record a massive amount of video footages as an online database. The author together with his colleagues and students have focused especially on broadcast news videos which are rich visual records of real-world phenomena. This paper reports the authors' works on the structuring, browsing, and the reusing of a large-scale news video archive: NII TV-RECS.

Historically, the first serious attempt on constructing an online broadcast news video archive started in 1994 as the Informedia [2] News-on-Demand [3] project at Carnegie Mellon University. News-on-Demand was a pioneering attempt that aimed for constructing a video archive system that continuously records video data broadcasted on TV from the air, and supports online access to the recorded footages by indexing and structuring them based on their contents.

In the following years, public institutions and the broadcasters themselves -mostly in Europe and in the USA - also started creating large-scale digital broadcast video archives including, but not limited to news videos. Especially, public institutions such as the Dutch Audio and Visual Institute (Nederlands In-

Received August 31, 2013; Accepted November 5, 2013.

ide@is.nagoya-u.ac.jp

This paper is a revised and translated version of the technical report [1] published as a hand-out for an invited talk at IEICE SIG PRMU on Feb. 2013

DOI: $10.2201 / \mathrm{NiiPi} .2014 .11 .3$ stitute voor Beeld en Geluid) ${ }^{1)}$ and the French National Audiovisual Institute (INA: Institut National de l'Audiovisuel $)^{2)}$ are not only large in their sizes and variety of contents, but also are leading a few steps ahead of the others in their styles of out-reach towards the public.

Meanwhile in the academic world, Dublin City University’s Físchlár-TV project (1999) [4] and the National Institute of Informatics's TV-RECS project (2001) [5] followed the Informedia project. Both projects have put special efforts on the handling of news videos over other contents ${ }^{3)}$. In addition, a video retrieval evaluation workshop TRECVID ${ }^{4), 5)}$ [7] started in 2001 which boosted both the advance of the field and the growth of the community. However, although the TRECVID dataset contained some news videos produced by several TV broadcasters around the World, the tasks were not defined from the point of news contents analysis, since that was not their main interest.

The works introduced in this paper are parts of the NII TV-RECS project. In the following sections, they will be introduced in two rough categories: 1) structuring and browsing, and 2) reusing.

\footnotetext{
1) http://www.beeldengeluid.nl/

2) http://www.ina.fr/

3) Namely, Físchlár-News [6], in the case of the former project.

4) http://trecvid.nist.gov/

5) It was originally a video track within the text retrieval evaluation workshop: TREC
} 
Table 1 Specifications of the NII TV-RECS news video archive (As of August 31, 2013).

\begin{tabular}{lr}
\hline Program & NHK “News 7" \\
\hline Period & 4,549 days \\
Number of records & 4,445 records \\
Length & Approximately 2,250 hours \\
Size (Closed-caption) & $104 \mathrm{MB}$ \\
Size (in MPEG-1 video) & $1.28 \mathrm{~TB}$ \\
Size (in MPEG-2 video) & $6.43 \mathrm{~TB}$ \\
\hline
\end{tabular}

\subsection{NII TV-RECS broadcast video archive}

Following such backgrounds, the author and his colleagues; Drs. Shin'ichi Satoh, Norio Katayama, and Hiroshi Mo, jointly created a broadcast video archive at the National Institute of Informatics, which started recording in March, 2001. The archive is composed of the following two sub-archives.

1. "24 hours video archive" which continuously and simultaneously records all seven terrestrial channels broadcasted in the Tokyo metropolitan area 24 hours a day. It is overwritten after a period of time.

2. "Particular programs archive" which records particular programs such as news, cook shows, and documentaries. It is never overwritten and preserved eternally.

These archives were later jointly named as the "NII TVRECS (NII TV Broadcast Video Research Corpus" ${ }^{\text {), }}$ and used for research purposes.

The works introduced in this paper made use of the "news video archive" from the particular program archive, which is a collection of videos from a single news program broadcasted daily. Table 1 shows the specification of this archive as of August 31, 2013.

After more than twelve years since the original system for analogue TV broadcasting (Fig. 1) was constructed, the current system is equipped with much larger storage space and has also been converted to receive digital TV broadcasting. Thus the details may be slightly obsolete, but the original system is introduced in reference [5].

\subsection{Definition of terms}

Terms related to news contents are defined as follows. First, the definition of basic terms by the TDT (Topic Detection and Tracking) Workshop organized by the National Institute of Standard and Technologies (NIST), whose target was to track topics in news arti-

\footnotetext{
6) http://www.vpl.nii.ac.jp/tv-recs/index-en.html
}

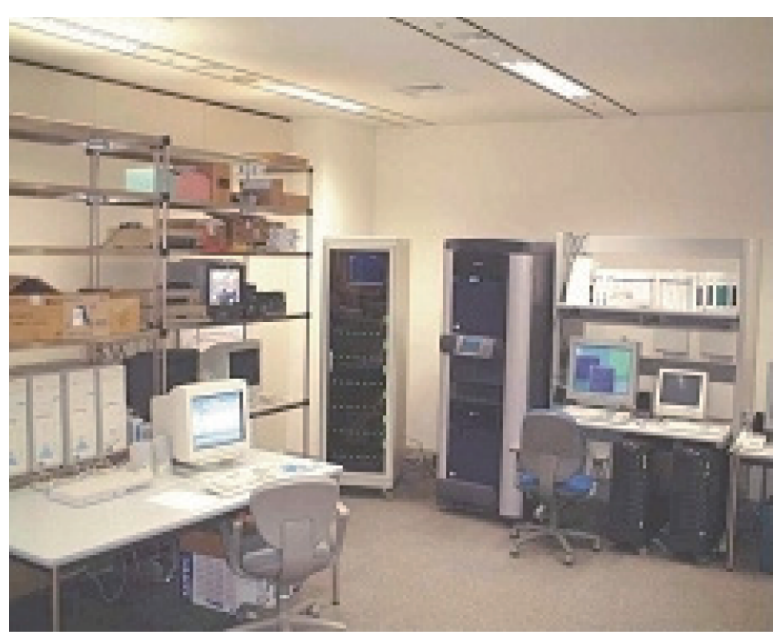

Fig. 1 The original NII TV-RECS video archive system.

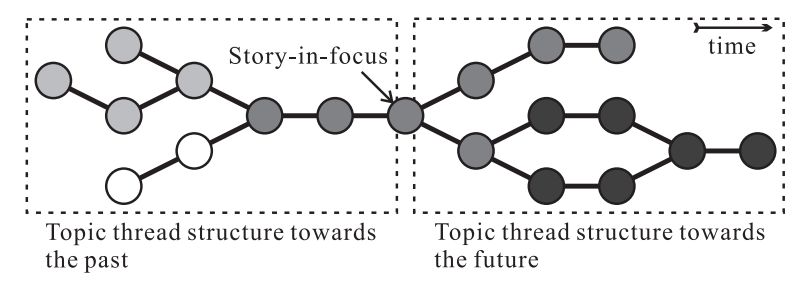

Fig. 2 Example of a topic thread structure: Each circular node represents a "story", and a set of stories painted in the same color forms a "topic cluster". A path that connects two arbitrary stories is a "topic thread".

cles, is as follows [8]:

- event: Some incident that occurred at some specific time and place along with all necessary preconditions and unavoidable consequences.

- story: A topically cohesive segment of news that includes two or more declarative independent clauses about a single event.

- topic: A seminal event or activity, along with all directly related events and activities.

In addition, the following concepts and terms were defined by the authors [9].

- topic thread: A sequence of related stories chained chronologically. It may contain several topics.

- topic thread structure: A directed graph composed of topic threads terminating / originating from a specified story. 


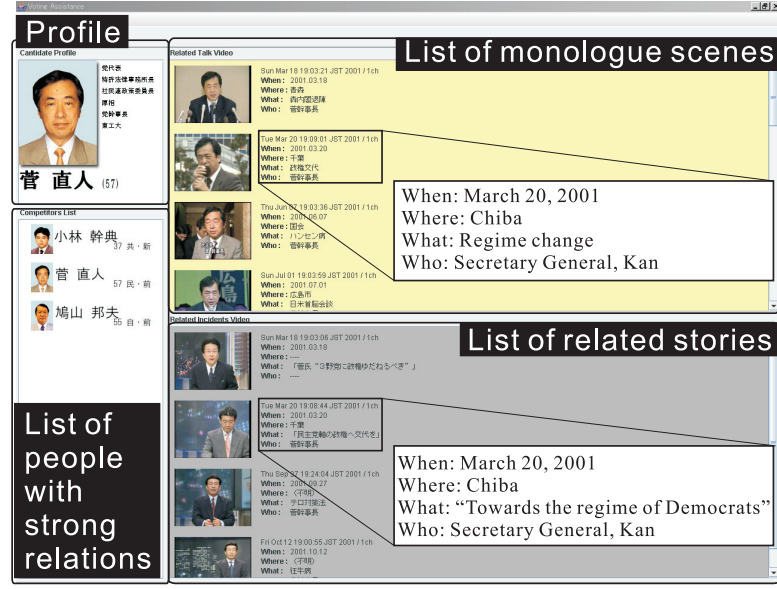

(a) Personal information view

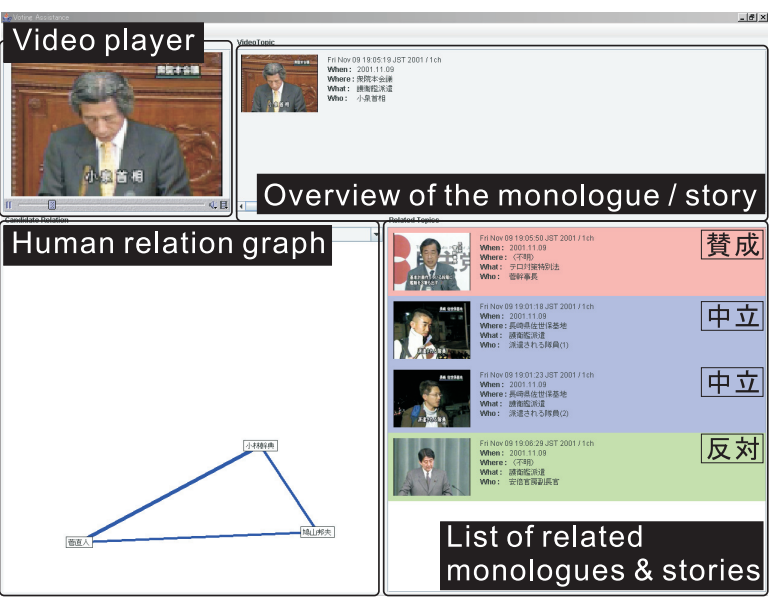

(b) Monologue / story view

Fig. 3 A prototype interface of a news people information portal: "NewsWho'sWho". Once a monologue or a story is selected in the personal information view, the view switches to the monologue / story view.

- topic cluster: A topically cohesive set of neighboring stories in the topic thread structure.

Figure 2 illustrates the concepts.

Note that most of the works introduced in this paper make use of audio transcript provided in the form of closed-caption (CC, hereafter). Also note that in this paper, "video" refers to multimedia information composed of "motion image", "audio", and "closedcaption".

\section{Structuring and browsing news videos}

When considering news contents in general, it is often said that the so-called " $5 \mathrm{~W} 1 \mathrm{H}$ " attributes are important. When considering the relation between (i.e. structuring) news contents, the more concrete " $4 \mathrm{~W}$ " (When, Where, Who, and What) are usually focused among these attributes. On the other hand, in some cases, the relations are considered in whole, where these attributes are not explicitly distinguished.

The works introduced here either focuses on the relation of the "Who" attribute (i.e. relation between people in the news) or the transition of topics by handling the relation in whole.

\subsection{Structuring and browsing according to people}

Although the archive system experienced various troubles in the beginning, after two years, in 2003, it was finally operating stably. Towards the end of the year, the group members gathered multiple times, and discussed the possible future applications of the archive.
Incidentally, the timing cooccurred with the 43rd General Election, so the members came up with the idea of supporting decisions for voting using the news video archive that contains rich multimedia reports on the activities of politicians such that text articles such as news papers and magazines can / do not necessarily inform the voters.

We then developed a prototype people information portal interface: "NewsWho'sWho" (Fig. 3). This interface was designed so that it could search events related to and statements by a specific politician, together with corresponding reactions (negative / neutral / positive) by other people. It also provided a visualization of social relations between people.

Although this interface was a prototype, and all the analyses and metadata were manually provided, it was a very clear and important goal for us to decide the direction of the project. As a matter of fact, all the works introduced in this section are technologies and their derivatives needed to automatically create the contents for this prototype interface.

\subsubsection{Browsing according to relations between people}

Just when we started focusing on people in news, research on social networks started boosting in surrounding research fields. Influenced by the concept, we developed an interface "trackThem" (Fig. 4).

This interface allowed users to explore human relations that were extracted according to their cooccurrence in stories in the news video archive. At the same time, the interface allowed the users to view video footages on news stories that a specified pair of persons were jointly involved. 


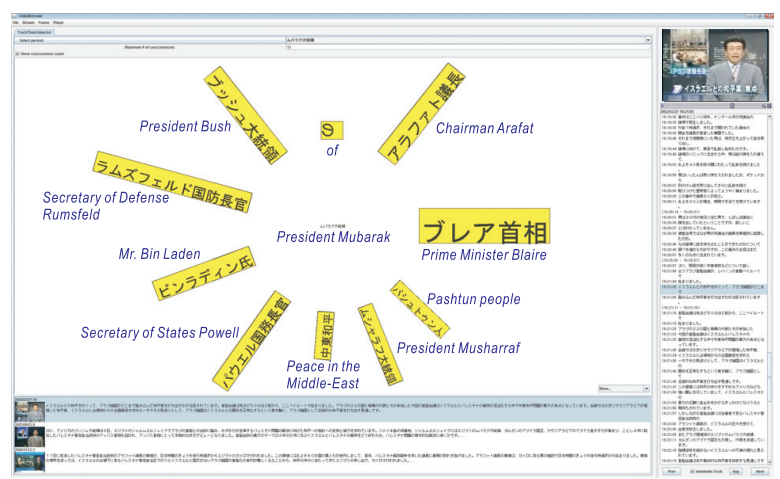

Fig. 4 A news video browsing interface based on the cooccurrence of people in news stories: "trackThem" [10]. People with strong relations with the person-in-focus are listed in a spiral form around him / her. The size of the names are scaled in proportion to the strength of relations. When the person on the spiral is clicked, a list of stories where the two persons cooccured appear at the bottom of the screen. Instead, if the person is double-clicked, the person becomes the person-in-focus in the center.

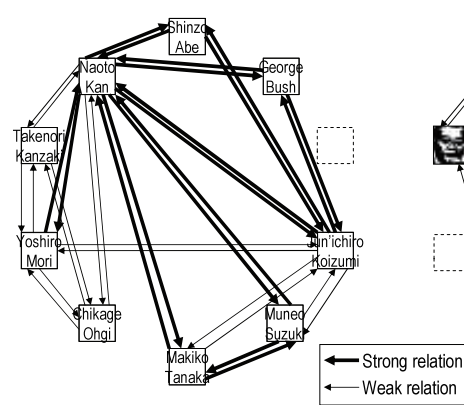

(a) Extracted from text

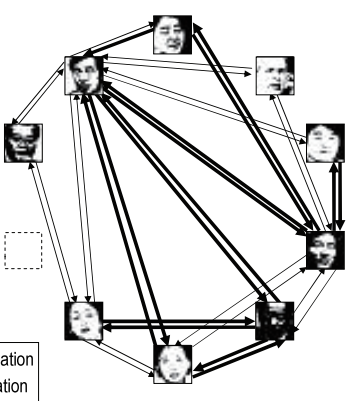

(b) Extracted from image
Fig. 5 Strength of cooccurrence-based relations of people extracted from news videos.

The original system was based on names that appeared in the $\mathrm{CC}$, but later we tried to extend the concept to the image-domain, hoping to extract unspoken (for various reasons) relations. As a matter of fact, as shown in Fig. 5, based on the actual data, we found the text-based and image-based cooccurrence being complementary, and confirmed that they should be combined. However, due to the difficulty in correctly labeling faces by image processing technologies, we did not succeed in actually implementing the image-based cooccurrence in the trackThem interface.

See reference [10] for details of the work.

\subsubsection{Name identification according to faces}

In order to solve the face labeling problem, we worked on the name disambiguation problem specific

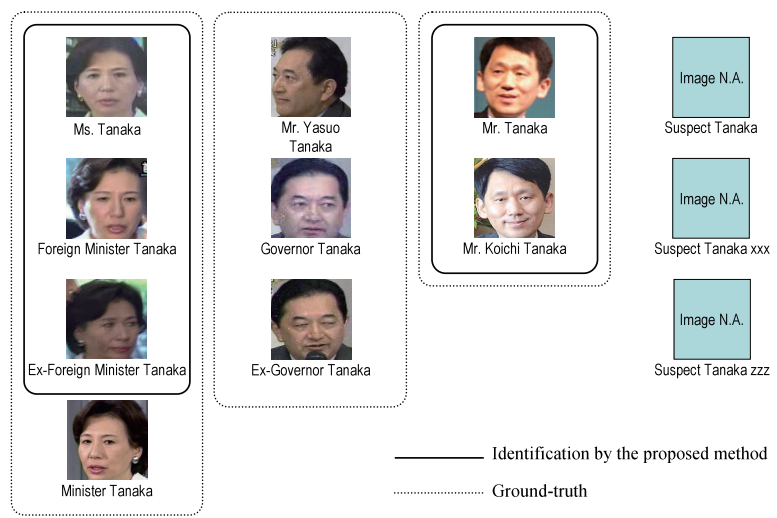

Fig. 6 An example of name disambiguation by faces (ex. "Tanaka") [11].

to news contents. The problem in news contents is that, the name and / or designation even for a same person can frequently change according to the topic and / or period, on top of the common problem that different people share the same name. Thus, we proposed a name identification method that combined both image and text information by comparing faces and names at the same time.

An example of the result obtained by the method is shown in Fig. 6. As it can be seen from the example, even without real-world knowledge, we could identify persons in news videos with different designations.

See reference [11] for details of the work.

\subsubsection{Detection of monologue scenes}

By the way, what can be the advantage of obtaining information on real-world phenomena from TV news instead of newspapers or magazine articles? If simply obtaining concrete facts about the event were the purpose, text-based media should be sufficient, or could even be better. - This had been a recurring question since the author started working with news video nearly twenty years ago. One answer to this question could be as the saying goes; "A picture is worth a thousand words". That is, there are so many undescribed multimedia information in news video such as the atmosphere or the condition that are / can not be described by text, and also pretty much subjective.

Considering this characteristic, we focused on detecting speeches, announcements, comments and so on in the form of a monologue from news videos, except for those by the anchor-person and the reporters. In order to do so, it was necessary to always confirm the synchronicity between the audio and the image channels, since in the case of news videos, the voice of an anchor-person or reporters quite often overlaps or even 
replaces the original speech.

Thus, we have developed a method that automatically learns and excludes voices of the anchorperson and the reporters according to specific keywords in the CC [12] and a method that learns the correlation of the features between the lip shape and the audio [13], in order to detect monologue scenes. See corresponding references for details of the works.

\subsection{Search and browsing according to topics}

As the size of the news video archive grew, we started noticing that simply listing video clips of stories that contain a query term in the traditional way was insufficient; as the list of results grew, even if they were mostly relevant to the query, it started becoming virtually infeasible to actually view them all in order to assess their relevance or to understand their contents due to the low perspicuity of video media. For example, if we input a popular name that had appeared in news for a long period, for example "Bin Laden", as a query, a traditional interface would return hundreds of stories that contained the name related to diverse topics such as terrorism, war, religion, and so on.

Moreover, due to the chronological characteristic of news contents, it can be easily assumed that users would actually wish to understand the transition or flow of the topic-of-interest, instead of understanding the details on individual stories. However, with the large amount of data, doing so comprehensively, especially for an arbitrary topic, is simply beyond human ability.

Thus, we first developed an algorithm that automatically creates a directed graph structure that represents the transition of topics called a "topic thread structure" by considering both the chronological and the semantic relation between stories, and next implemented a news video browsing interface named "mediaWalker", that visualizes the structure and also allows users to actually browse the video along the structure (Fig. 7).

The following three works introduced in this section are parts that compose or extend the interface.

\subsubsection{Extraction of a chronological semantic structure of topics: "topic thread structure"}

In order to create a structure that represents the transition of topics, the traditional solution was to simply cluster the stories according to their contents (topic clustering) and then connected them linearly in chronological order. A more complex structure that can handle the transition of multiple topics can be represented as a directed tree, by considering both the chronological and the semantic relations between stories. However, the tree structure could not compactly represent the divergence and the reunion of the topic transition.

Thus, we developed an algorithm that could repre-

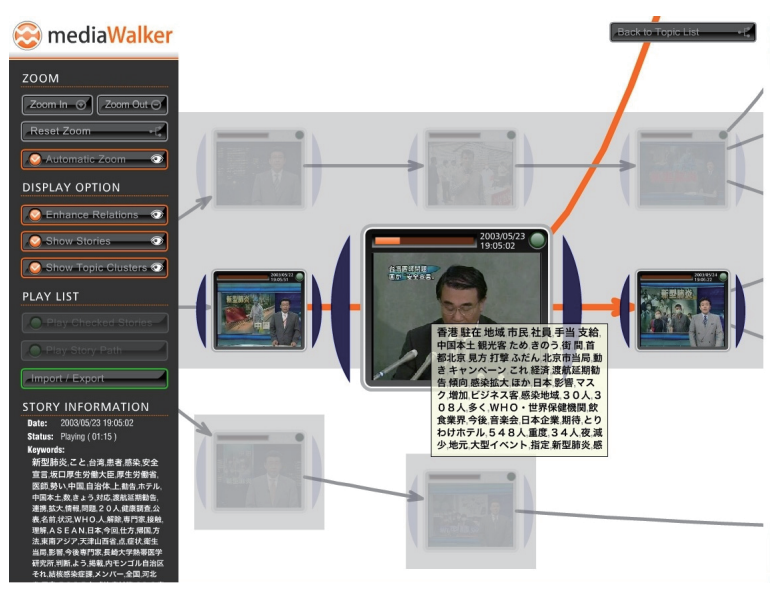

Fig. 7 A news video browsing interface based on the topic thread structure: "mediaWalker" [9]. The users can watch the actual video while exploring the transition of topics along a topic thread structure.

sent the divergence and the reunion of the topic transition by considering both the chronological and the semantic relations between stories, and also by representing the structure as a directed graph, namely a "topic thread structure".

The mediaWalker interface is an interface that visualizes the topic thread structure starting from an arbitrary specified story, and allows the users to actually browse corresponding video clips of stories along the structure.

See reference [9] for details of the work.

\subsubsection{Multimedization of Wikipedia articles}

The topic threading algorithm and the original mediaWalker interface expected as an input, video clips of stories from a specific news program broadcasted once a day. In order to introduce related contents from other sources including other news programs and Web documents to the framework, one approach is to simply define some kinds of relation metrics and apply the same algorithm to them. Although this should work in theory, it was easy to imagine that the topic thread structure would become unnecessarily complicated and redundant, since there would be many very similar stories discussing the same event at a very close timing.

Thus, we decided to consider the topic thread structure obtained from the NII TV-RECS as a backbone, and then add links from each story to external resources.

One example taking this approach is the automatic linking to the user-contributed online encyclopedia "Wikipedia"7) that started booming then. Considering

\footnotetext{
7) http://www.wikipedia.org/
} 


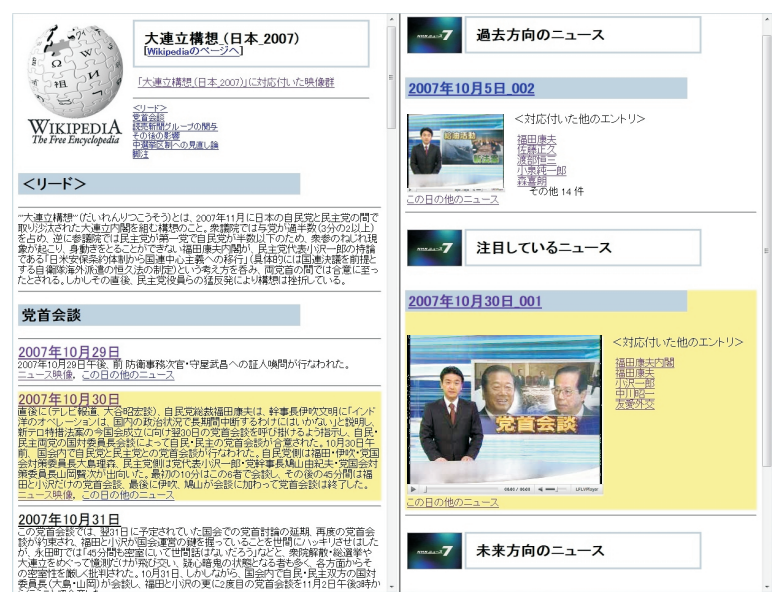

Fig. 8 A news video browsing interface linked to Wikipedia articles: "Videopedia" [14]. The left-side panel shows the timeline extracted from a Wikipedia article, and the rightside panel shows a video clip of the story corresponding to the selected description and neighboring stories along the topic thread structure.

the characteristics of news contents, before comparing the relation between text contents, we selected candidates of news stories that could possibly be linked to a Wikipedia article by comparing the broadcasted date and the date expressions in the article.

From the other way around, this process could be considered as automatic "multimedization" of Wikipedia articles by linking corresponding video clips to the mostly text articles. We have actually implemented this as an interface named "Videopedia" (Fig. 8).

See reference [14] for details of the work.

\subsubsection{Cross-lingual news story retrieval}

Another example is the automatic linking to other news programs, especially to those broadcasted in other countries in other languages. This could be useful to understand the event from diverse aspects by viewing how the same event is introduced in other countries. Traditionally, this task had been challenged through text-based information retrieval methods, but they could not handle cases that the view-points were extremely different and thus very few texts cooccurred. In order to handle such cases, we decided to make use of the cooccurrence of image information in addition to that of text information.

Concretely, we made use of a near-duplicate video clip detection method that we had developed [15], and detected stories that contained the same event broadcasted in different channels, especially in different countries and in different languages, in combination

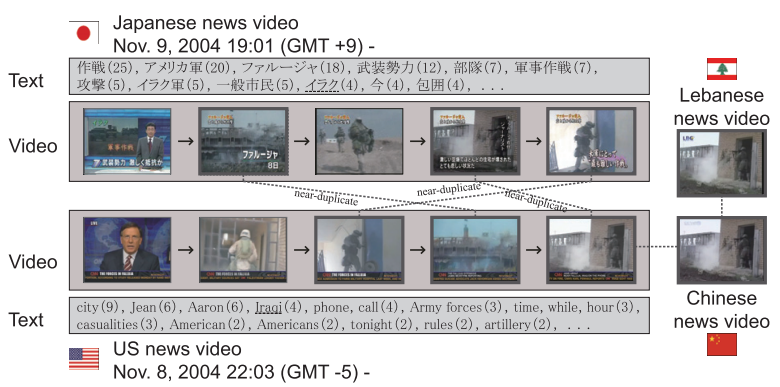

Fig. 9 Example of stories broadcasted in different countries associated according to the cooccurrence of nearduplicate video clips[16]. Only the underlind terms matched in the text, where three near-duplicate video segements were found.

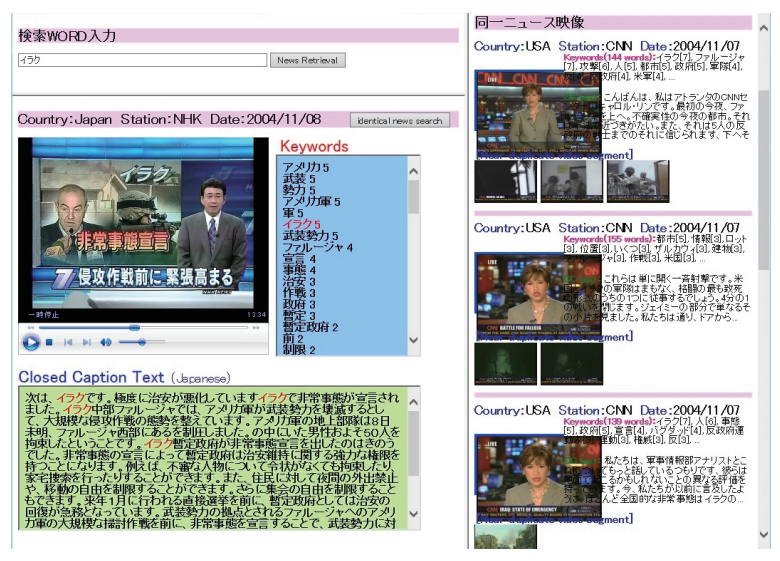

Fig. 10 Cross-lingual news story browsing interface: "mediaTraveller" [16]. Stories closely related to the story infocus shown in the left-side panel are listed on the rightside panel. The small thumbnails are the near-duplicate video clips that cooccured in both stories.

with machine-translated text cooccurrences. Figure 9 shows an example where a related story was successfully detected although the number of cooccurrence in the text was very small, since the number of nearduplicate video segments was large enough.

In addition, as a traditional query-based search interface, we implemented a cross-lingual story retrieval interface: "mediaTraveller" as shown in Fig. 10.

See reference [16] for details of the work.

\section{Reusing news videos}

As introduced in the previous sections, we had worked on the structuring and the browsing of a largescale news video archive. However, since around 2008, when the size of the archive surpassed 1,000 hours in video length, we started to notice that the archive could 


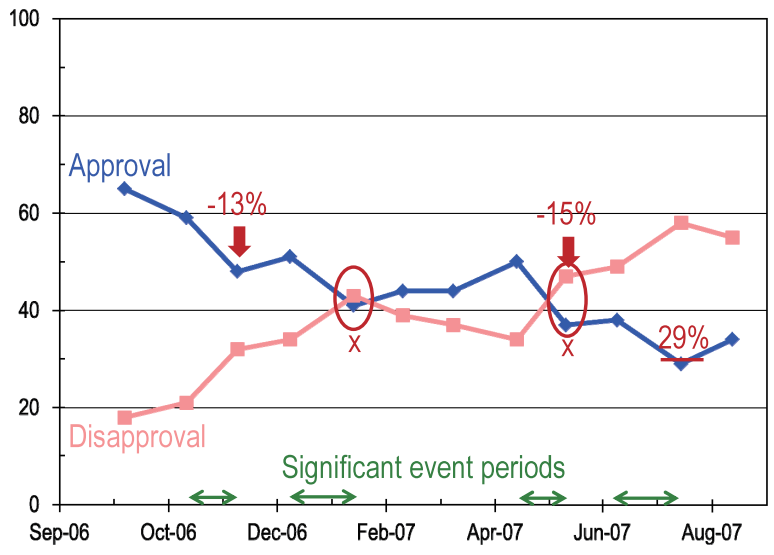

Fig. 11 Transition of demoscopic polls and major events (ex-Prime Minister Abe's first government) [17].

be considered as a large multimedia resource on realworld phenomena. Since then, our target has slowly shifted from the analysis itself towards the generation of new contents using the analyzed results. As a model or a "scenario" needed to generate the new contents, we have been making use of related contents obtained from external sources. We call this approach, "video story telling".

One example of the approach is our work on explaining a personal history using the news video archive. The original idea was to automatically generate an obituary video, since an unexpected death of a person requires a TV broadcaster to rapidly generate a summary video on his / her life in order to broadcast it in the earliest occasion. However, since our archive only covers slightly more than ten years, its coverage was far too short to cover a normal person's lifetime. In addition, in most cases, even very famous people are not broadcasted in news throughout their lifetimes. Thus, we decided to work on the case of a politician's professional life, concretely, a Japanese Prime Minister's period in his office; from inauguration to resignation, and explain why he had to resign in the end.

As an external source, the monthly demoscopic polls (approval and disapproval rates) of the government ${ }^{8)}$ was referred to, and their characteristic behaviors (absolute values (high / low), trend (increase / decrease), and inversion of approval and disapproval) were used to detect significant event periods (months) (Fig. 11). Then, stories that contain the actual events that caused the characteristic behaviors were estimated by referring to the topic thread structures originating from each

8) The "Monthly Survey on Political Attitude" published monthly in The Monthly Report on Broadcast Research by NHK Broadcasting Culture Research Institute was used as the source.

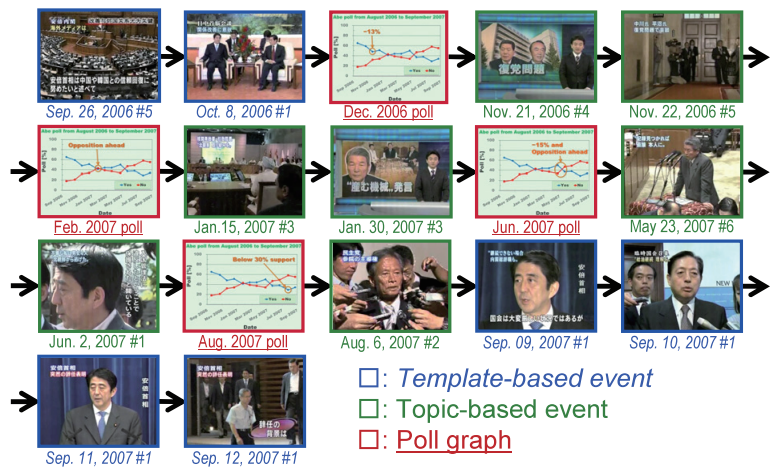

Fig. 12 A summary video that explains why the Prime Minister resigned (ex-Prime Minister Abe's first government) [17].

story during the significant event periods; a story that is a beginning or an end of a topic thread structure, or a story with a high density of stories in the topic thread structure was considered as event candidates. Finally, the event candidate stories were ranked and selected according to sentiment analysis on the CC.

As for the editing part, video clips that contain the selected stories and CG clips showing the poll graph with indications on its characteristic behaviors were concatenated to generate a summary video. Figure 12 shows an example of the generated summary video.

See reference [17] for details of the work.

\section{Summary}

In this paper, the author has reported works on the analyses and the applications of a large-scale news video archive in the past twelve years and more. He is currently working towards the next step of the new contents generation using the video contents in the archive by incorporating real-world information available on the Internet, especially social networking services such as Twitter and Facebook, in order to reflect the opinion of general users in the generation process.

\section{Acknowledgement}

The works introduced in this paper were mostly done at the National Institute of Informatics, Nagoya University, and University of Amsterdam with the following colleagues and students (in alphabetic order): Daisuke Deguchi, Keisuke Doman, Norio Katayama, Tomoyoshi Kinoshita, Takashi Kobayashi, Shogo Kumagai, Hiroshi Mo, Hiroshi Murase, Frank Nack, Kazuhiro Noda, Takashi Ogasawara, Akira Ogawa, Tomoki Okuoka, Shin'ichi Satoh, Naoki Sekioka, Yuji Shamoto, and Tomokazu Takahashi.

Parts of the works introduced in this paper were 
supported by the following fundings: JSPS/MEXT Grants-in-Aid for Scientific Research on Priority Areas "Informatics" (13224093, 15017285, 16016289) and "Infoplosion" (18049035, 19024036, 21700103), Grants-in-Aid for Young Scientists (B) (15700116, 18700080, 21013022), Grant-in-Aid for Scientific Research (B) (23300036), Nagoya University Information Science 21st Century COE Program, research grants from Kayamori Foundation of Information Science Advancement (K17KenX-202), and Hoso Bunka Foundation (211033), joint research projects with the National Institute of Informatics and Toshiba Corporate R\&D Center, contracted research with Mitsubishi Research Institute, Inc., Excellent Young Researcher Overseas Visit Program (21-5324) from JSPS.

\section{References}

[1] I. Ide, "[Special Talk] Analysis and Applications of a Large-Scale News Video Archive (in Japanese)," Technical Report of IEICE, no. PRMU2012-138, p.6, 2013.

[2] H. Wactlar, T. Kanade, M. Smith, and S. Stevens, "Intelligent Access to Digital Video: The Informedia Project," IEEE Computer, vol.29, no.5, pp.46-52, 1996.

[3] A. G. Hauptmann, M. J. Witbrock, and M. G. Christel, "News-on-Demand: An Application of Informedia Technology," D-Lib Magazine, vol.1, no.3, Online, 1995.

[4] A. F. Smeaton, H. Lee, and K. McDonald, "Experiences of Creating Four Video Library Collections with the Físchlár System," International Journal on Digital Libraries, vol.4, no.1, pp.42-44, 2004.

[5] N. Katayama, H. Mo, I. Ide, and S. Satoh, "Mining Large-Scale Broadcast Video Archives towards Inter-Video Structuring," Advances in Multimedia Information Processing, PCM2004, Fifth Pacific Rim Conference on Multimedia, Tokyo, Japan, Nov./Dec. 2004, Proceedings Part II, Lecture Notes in Computer Science, Berlin, Heidelberg, vol.3332, Edited by K. Aizawa, Y. Nakamura, and S. Satoh, SpringerVerlag, pp.489-496, 2004.

[6] H. Lee, A. F. Smeaton, N. O'Connor, and B. Smyth, "User Evaluation of Físchlár-News: An Automatic Broadcast News Delivery System," ACM Transactions on Information Systems, vol.24, no.2, pp.145-189, 2006

[7] A. F. Smeaton, P. Over, and W. Kraaij, "Evaluation Campaigns and TRECVid," Proceedings of the Eighth ACM International Workshop on Multimedia Information Retrieval (MIR'06), Boston, Dordrecht, London, pp.321-330, Oct. 2006

[8] J. G. Fiscus and G. R. Doddington, "Topic Detection and Tracking Evaluation Overview," Topic Detection and Tracking: Event-Based Information Organization, Boston, Dordrecht, London, Edited by J. Allan, Kluwer Academic Publishers, pp.17-31, 2002.
[9] I. Ide, T. Kinoshita, T. Takahashi, H. Mo, N. Katayama, S. Satoh, and H. Murase, "Efficient Tracking of News Topics Based on Chronological Semantic Structures in a Large-Scale News Video Archive," IEICE Transactions on Information and Systems, vol.E95-D, no.5, pp.12881300, 2012.

[10] I. Ide, T. Kinoshita, H. Mo, N. Katayama, and S. Satoh, "trackThem: Exploring a Large-Scale News Video Archive by Tracking Human Relations," Information Retrieval Technology, Second Asia Information Retrieval Symposium, AIRS2005, Jeju Island, Korea, Oct. 2005 Proceedings, Lecture Notes in Computer Science, Berlin, Heidelberg, vol.3689, Edited by G.G. Lee, A. Yamada, H. Meng, and S.H. Myaeng, SpringerVerlag, pp.510-515, 2005.

[11] I. Ide, T. Ogasawara, T. Takahashi, and H. Murase, "Name Identification of People in News Video by Face Matching," Proceedings of the Third International Workshop on Computer Vision meets Databases (CVDB'07), pp.17-21, June 2007.

[12] I. Ide, N. Sekioka, T. Takahashi, and H. Murase, "Assembling Personal Speech Collections by Monologue Scene Detection from a News Video Archive," Proceedings of the Eighth ACM SIGMM International Workshop on Multimedia Information Retrieval (MIR'06), pp.223-229, Oct. 2006.

[13] S. Kumagai, K. Doman, T. Takahashi, D. Deguchi, I. Ide, and H. Murase, "Speech Shot Extraction from Broadcast News Videos," International Journal of Semantic Computing, vol.6, no.2, pp.179-204, 2012.

[14] T. Okuoka, T. Takahashi, D. Deguchi, I. Ide, and H. Murase, "Labeling News Topic Threads with Wikipedia Entries," Proceedings of the Eleventh IEEE International Symposium on Multimedia (ISM'09), pp.501-504, Dec. 2009

[15] I. Ide, K. Noda, T. Takahashi, and H. Murase, "GenreAdaptive Near-Duplicate Video Segment Detection," Proceedings of the 2007 IEEE International Conference on Multimedia and Expo (ICME'07), pp.484-487, July 2007.

[16] A. Ogawa, T. Takahashi, I. Ide, and H. Murase, "CrossLingual Retrieval of Identical News Events by NearDuplicate Video Segment Detection," Advances in Multimedia Modeling, Fourteenth International Multimedia Modeling Conf., MMM2008, Kyoto, Japan, Jan. 911, 2008 Proceedings, Lecture Notes in Computer Science, Berlin, Heidelberg, vol.4903, Edited by S. Satoh, F. Nack, and M. Etoh, Springer-Verlag, pp.287-296, 2008 .

[17] I. Ide and F. Nack, "Explain This to Me! —A Study on Automatic Recompilation of Broadcast News Video-," ITE Transactions on Media Technology and Applications, vol.67, no.2, pp.101-117, 2013. 


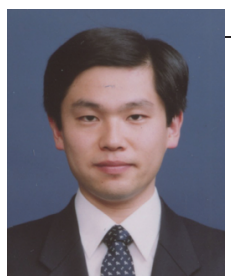

\section{Ichiro IDE}

Dr. Ichiro IDE received his Ph.D. in 2000 from The University of Tokyo, Japan. He has worked as an Assistant Professor at the National Institute of Informatics, Tokyo, Japan, during 2000 and 2004. He is currently an Associate Professor at Nagoya University, Japan. He was an Invited Professor at IRISA, France in 2005, 2006, and 2007, a Senior Visiting Researcher at University of Amsterdam, the Netherlands from 2010 to 2011. He is a member of IEEE, ACM, IEICE, IPS Japan, ITE, JSAI, and ANLP. 